\title{
APROXIMACIÓN AL SERVICIO DE ASISTENCIA PERSONAL Y SU IMPLANTACIÓN EN ESPAÑA. PERFIL DEL BENEFICIARIO DE LA PRESTACIÓN EN GALICIA
}

\author{
An approach to personal assistance service and its implementation in \\ Spain. The profile of the beneficiary of the provision in Galicia
}

Rubén GonzÁlez-Rodríguez ${ }^{1}$, Manuel Gandoy Crego ${ }^{2}$, CARMen Verde Diego $^{3}$

\section{Resumen}

Introducción: El objetivo de esta investigación es conocer y estudiar el perfil de la persona beneficiaria de la prestación de asistencia personal, una de las prestaciones del Catálogo de servicios y prestaciones del Sistema para la Autonomía y Atención a la Dependencia.

Material y métodos: Mediante un estudio observacional descriptivo de prevalencia, se persigue conocer el perfil de las personas beneficiarias de la prestación de asistencia personal y el grado de implantación de la misma.

Resultados: Los datos nos muestran que la prestación económica de asistencia personal sigue siendo, dentro del catálogo de servicios y prestaciones del Sistema, una de las modalidades de atención de menor resolución en Galicia y en el conjunto del Estado.

Discusión: La figura de asistente personal no ha conseguido establecerse, hasta el momento, como un servicio de referencia para las personas con diversidad funcional. En el presente trabajo identificamos una serie de factores que entendemos dificultan el desarrollo de esta prestación y la implantación efectiva de la misma.

\begin{abstract}
Introduction: The main goal of this research is to know and study the profile of the person who receives the economic benefit of personal assistance, one of the compensations in the Catalogue of Services and Benefits of the System for Autonomy and Attention of Dependents.

Material and Methods: With a descriptive study of prevalence, this study seeks to understand the profile of the beneficiaries of personal assistance and its degree of implementation.

Results: The data shows that the economic support for personal assistance remains, within the Catalogue of Services and Benefits of the System, one of the modalities of care with lower resolutions in Galicia and in the whole State.

Discussion: The figure of personal assistant has not been established, so far, as a referral service for people with functional diversity. In the present article we identified a series of factors that hinder the development of this provision and the effective implementation of the same.
\end{abstract}

Keywords: Dependency; disabled persons; personal autonomy; personal assistant; Galicia.

Palabras clave: Dependencia; personas con discapacidad; autonomía personal; asistente personal; Galicia.

1. Universidad de Santiago de Compostela (USC), Escuela Universitaria de Trabajo Social, A Coruña, España. Servicio de Dependencia y Autonomía Personal. C/ Sáenz Díez, 33 bajo - 32003 Ourense.Teléfono: 653480443. E-mail: rgonzalez@euts.es / ruben.gonzalez.rodriguez@xunta.es

2. Universidad de Santiago de Compostela (USC), Departamento de Enfermería, A Coruña, España.

3. Universidad de Vigo (UVigo), Departamento de Análisis e intervención psicosocioeducativa, Ourense, España. 


\section{Introducción}

La preocupación por las personas «diferentes» y las conductas «anormales» ha sido una constante histórica en todas las civilizaciones. Considerando que las discapacidades tienen lugar en un contexto social, económico y político concreto, es verdad que la comprensión y las actitudes que la sociedad ha manifestado hacia las personas con discapacidad no siempre han sido las mismas (Medina, 2010). Sin embargo, podemos reparar que «en el tratamiento dado a los deficientes ${ }^{4}$ hay variaciones históricas entre épocas y entre culturas que, de forma muy sintetizada, giran en torno a una constante histórica, la marginación» (Aguado, 1995: 26).

Mucho se ha avanzado en los últimos años en la implantación de políticas sociales que den protección a las personas con discapacidad y eviten (o frenen) esas situaciones de marginación y desigualdad a las que se han visto sometidas a lo largo de la historia. El contenido, y el desarrollo, de algunas de estas políticas viene derivado del empuje suscitado por movimientos sociales de la ciudadanía. Un ejemplo de ellos es el Movimiento de vida independiente.

El Movimiento de vida independiente es un movimiento social que nació en los años 60, en Estados Unidos (Berkeley), para defender los derechos civiles de las personas con discapacidad y luchar por su emancipación y empoderamiento. Acostumbra tomarse como fecha de inicio de esta corriente el año 1962, cuando Ed Roberts, afectado por una discapacidad severa, consigue ser admitido en la Universidad de California (García, 2003).

En España, la discapacidad está protegida en la norma de mayor rango en nuestro ordenamiento jurídico, la Constitución Española de 1978. Así, en su artículo 49, se establece que los poderes públicos realizarán una política de previsión, tratamiento, rehabilitación e integración de los disminuidos físicos, sensoriales y psíquicos, a los que prestarán la atención especializada que requieran y los ampararán especialmente para el disfrute de los derechos que (...) otorga a todos los ciudadanos.

Además de la normativa interna, la legislación del Estado se nutre de los tratados y acuerdos internacionales ratificados por España. En materia de discapacidad queremos destacar la Convención sobre los derechos de las personas con discapacidad aprobada por la Asamblea de la ONU el 13 de diciembre de 2006 (United Nations, 2006). Nuestro país firmó dicha Convención y el protocolo facultativo adicional el 30 de marzo de 2007 y el 30 de diciembre del mismo año ratificó su adhesión. Desde ese momento, las disposiciones

4. Se mantiene la expresión deficiente por tratarse de una cita literal del autor. Como veremos a lo largo de este trabajo, actualmente este término está en desuso. 
contenidas en la Convención, forman parte del ordenamiento jurídico español (arts. 10.2 y 93-96, Constitución Española).

En España, no se crea un sistema de protección específico para atender las situaciones de dependencia hasta el año 2007. Previamente, la protección a las personas mayores y/o con discapacidad que precisaban de la asistencia de otra persona se hacia desde otros sistemas de protección social ya existentes. Sempere (2008) concentra estas atenciones en dos grandes áreas: 1) la preexistente protección a la dependencia desde la Seguridad Social y 2) la preexistente protección a la dependencia desde los Servicios sociales y de salud.

A través de la Ley 39/2006, de 14 de diciembre, de promoción de la autonomía personal y atención a las personas en situación de dependencia (en adelante LAPAD), se garantiza el derecho subjetivo de la ciudadanía a ser atendidos cuando se reconozca, por el organismo competente, una situación de dependencia (entre otros requisitos). El instrumento para llevar a cabo el desarrollo de este derecho es el Sistema para la Autonomía y Atención a la Dependencia (en adelante SAAD), que se configura como una red de utilización pública que integra, de forma coordinada, centros y servicios, tanto públicos como privados.

La medición de la situación de dependencia se estableció en tres categorías en función de su gravedad. El Baremo de valoración de dependencia recoge la clasificación de la situación de dependencia en tres grados de severidad: (en orden descendente) la gran dependencia (grado III), la dependencia severa (grado II) y la dependencia moderada (grado I). Además, dispone dos niveles en cada uno de los grados ${ }^{5}$, en función de la autonomía de la persona, así como de la intensidad de apoyo que requiere.

El SAAD posibilita que las personas en situación de dependencia puedan acceder a las prestaciones recogidas en su catálogo ${ }^{6}$. Siguiendo la clasificación establecida por Luján, Rodríguez y Fernández (2008), podemos considerar que las prestaciones incluidas en el catálogo del SAAD pueden tener, por su contenido, las características de prestaciones en especie o mediante servicios,

5. Los niveles de dependencia fueron suprimidos por el Real Decreto-ley 20/2012, de 13 de julio. Sin embargo, no determina que la Administración deba revisar de oficio las resoluciones de reconocimiento de la situación de dependencia por lo que muchos de los beneficiarios conservan un reconocimiento en el que se recoge tanto el grado como el nivel de dependencia.

6. La disposición final primera de la LAPAD (modificada por el Real Decreto-ley, de 13 de julio) establece que la efectividad del derecho a las prestaciones del SAAD se ejercitará progresivamente priorizando el acceso de las personas con mayor grado de dependencia reconocida. Según la normativa vigente en la actualidad los últimos grados en acceder al sistema lo harán el 1 de julio de 2015. 
o bien prestaciones de tipo económico (bien de tracto único o de tracto sucesivo). Pues bien, en el Catálogo de servicios y prestaciones del SAAD se incluye por primera vez, como prestación pública, la Asistencia personal, uno de los servicios de atención para personas con discapacidad que la propia Convención sobre los derechos de las personas con discapacidad (United Nations, 2006) inspiraba a potenciar entre los estados adheridos a la misma.

Inicialmente, de la prestación de asistencia personal solamente podían beneficiarse aquellas personas a las que se le hubiera reconocido una situación de gran dependencia, pero en la actualidad se garantiza esta atención independientemente del grado reconocido (art. 22 siete, Real Decreto-ley 20/2012).

La Asistencia Personal es un servicio de apoyo a las personas con diversidad funcional ${ }^{7}$, no desarrollado en el ámbito de los servicios sociales de España hasta hace poco, pero que lleva más de 30 años establecido en países como EE.UU., Suecia, Gran Bretaña, Noruega, Brasil, etc. (Rueda, 2013) y muy vinculada al Movimiento de vida independiente. Rodríguez-Picavea (2007: 117) define al asistente personal como «aquella persona que ayuda a otra a desarrollar su vida, esto es, que realiza o ayuda a realizar las tareas de la vida diaria a otra persona que, por su situación, bien sea por una diversidad funcional, o por otros motivos, no puede realizarlas por sí misma».

Al respecto de las tareas que desarrolla un asistente personal, siguiendo a Rodríguez-Picavea y Romanach (2006) podemos dividirlas y agruparlas en diferentes áreas: 1) tareas personales; 2) tareas del hogar; 3) tareas de acompañamiento; 4) tareas de conducción; 5) tareas de comunicación; 6) tareas de coordinación; 7) tareas excepcionales; y 8) tareas especiales. De la denominación de la mayor parte de las áreas puede desprenderse claramente las tareas que tienen asociadas, por ello queremos destacar las tres últimas.

7. Nos parece importante en este punto aclarar una cuestión conceptual. Entendemos, al igual que Campo, Crespo y Verdugo (2003: 21) que una de las mejores formas de estudiar la terminología utilizada es mediante el análisis de la redacción normativa y jurisprudencia desarrolladas en materia de discapacidad. En España, los términos minusválidos y personas con minusvalía han sido substituidos muy recientemente por persona con discapacidad desde la entrada en vigor de la LAPAD. Pasa a tomarse como criterio (además de realizar un proceso de sustantivación) lo recogido en la Clasificación Internacional del Funcionamiento, la Discapacidad y la Salud (Organización Mundial de la Salud, 2001) que adopta el término discapacidad como un archilexema que engloba la deficiencia, las limitaciones en la actividad y las restricciones en la participación (Casado, 2001: 7). En los últimos años se ha seguido avanzando en la cuestión terminológica y el Foro de vida independiente propone la expresión persona con diversidad funcional para designar lo que se conoce como discapacidad. Con este término se pretende eliminar las connotaciones negativas, y reforzar la esencia de diversidad (Iáñez, 2009). 
Las tareas de coordinación son las referidas a la planificación del día a día y a la ayuda de toma de decisiones. Las tareas excepcionales son aquellas intervenciones que vienen provocadas por una crisis de la persona asistida (que puede ser de carácter físico o psíquico). Resaltamos estos grupos de tareas ya que la asistencia personal acostumbra a vincularse solo con las limitaciones de tipo físico o sensorial. Por el contrario, esta prestación también es adecuada para personas con diversidad funcional psíquica y/o intelectual para realizar tareas de apoyo en la toma de decisiones y a la estructuración de la vida diaria.

Las tareas especiales son las referidas a actividades relacionadas con las actividades sexuales, en todo lo concerniente al acompañamiento y preparación. Acentuamos este último grupo de tareas ya que, si el concepto de asistencia personal es relativamente nuevo en el ámbito asistencial de las personas con diversidad funcional (al menos en nuestro país), más lo es el disponer de un servicio de asistencia para intervenir en esta esfera tan íntima de la persona.

García (1990) afirmaba en los años noventa que «la sexualidad de la persona disminuida, ya de por sí marginada, será mucho menos comprendida y más reprimida que las del ciudadano normal (...)». Sin duda, esta marginación perdura en la actualidad en las personas con diversidad funcional principalmente asociada a otra dificultad, el hecho de convivir permanentemente con sus progenitores o bien estar institucionalizados en equipamientos de tipo residencial (Arnau, 2004).

A diferencia de España, muchos países de nuestro entorno más próximo ya han regulado normativamente las tareas del Asistente personal e incluso han ido un paso más allá. En Holanda, Dinamarca, Alemania o Suiza aparece, vinculada a la atención de este tipo de necesidades, la figura del Acompañante sexual para personas con discapacidad que, dentro de un marco donde están definidas una serie de prestaciones, realiza un acompañamiento adecuado en función de las emociones y los deseos sensoriales y eróticos de los beneficiarios («Sexualité», 2010).

El servicio de asistencia personal es, dentro del SAAD, una prestación económica de tracto sucesivo, cuya finalidad es promocionar la autonomía de las personas en situación de dependencia, en cualquiera de sus grados. Su objetivo es contribuir a la contratación de una asistencia personal, durante un número de horas, que facilite al beneficiario el acceso a la educación y al trabajo, así como una vida más autónoma en el ejercicio de las actividades básicas de la vida diaria (en adelante ABVD) (art. 19, LAPAD). 
Objetivos de la investigación

En base a nuestros planteamientos teóricos, con esta investigación pretendemos establecer si el objetivo de promocionar la autonomía que se recogía en el propio título de la LAPAD se está llevando a cabo en el marco del SAAD. Para ello focalizaremos nuestra atención en la implantación actual de la Prestación económica de asistencia personal (en adelante PEAP). Establecemos como objetivo específico conocer cuantas personas se benefician de la PEAP, tanto en el conjunto del Estado como en Galicia. Del mismo modo, estudiaremos el perfil de las personas beneficiarias de esta prestación en la comunidad autónoma de Galicia. Entendemos necesario este estudio previo, para poder comprender porqué es una de las prestaciones con menor índice de resolución dentro del Sistema para la Autonomía y Atención a la Dependencia en España.

\section{Material y métodos}

El tipo de estudio llevado a cabo es un estudio observacional descriptivo de prevalencia. Se desenvuelve en al ámbito de la comunidad autónoma de Galicia, y la recogida y explotación de datos se realizó entre los meses de noviembre de 2013 y enero de 2014.

La población objeto de estudio la constituyen aquellos solicitantes de valoración de dependencia a los que en su Programa individual de atención se les reconoció como modalidad de atención idónea la prestación económica de asistencia personal.

En la investigación que presentamos no procede selección muestral, puesto que se analizaran los datos de la totalidad de usuarios a los que se ha reconocido este tipo de prestación desde la entrada en vigor de la LAPAD el 01 de enero de 2007 hasta el 01 de octubre de 2013. Teniendo en cuenta este criterio de inclusión, se obtiene una población de 63 sujetos.

Respecto a las variables analizadas, divididas en sus diferentes categorías, se encontrarían las que siguen:

- Datos personales. Dentro de este grupo se encontrarían las variables edad y sexo. Los rangos en los que se agrupan los datos por edades fueron aglutinados, a criterio de los autores, en cinco grandes grupos: las personas menores de edad; las personas de 65 o más años; y tres rangos intermedios, de 18 a 35 años, de 36 a 50 años y de 51 a 64 años.

- Datos sociodemográficos. Referente a esta tipología se recogen las siguientes variables: provincia de residencia y número de habitantes de la localidad de residencia. Para poder agrupar los datos se han 
respetado los rangos de población que establece el Instituto Gallego de Estadística (IGE) en el censo de población y viviendas correspondiente al año $2011^{8}$.

- Datos sobre la situación de dependencia. En esta tipología se recogen: el grado y nivel de dependencia reconocido, el año de resolución del grado de dependencia, el año de resolución del Programa individual de atención y el tipo de tabla del Baremo de valoración de dependencia.

Para finalizar este apartado, pasamos a describir el procedimiento utilizado. Todos los datos de cada una de las variables descritas (todas las disponibles informatizadas) fueron obtenidos de la aplicación informática del Sistema Gallego de Atención a la Dependencia (SIGAD). Este proceso se realizó con la oportuna autorización previa de la Subdirección General de la dependencia (Xunta de Galicia), garantizando por parte de los investigadores la absoluta confidencialidad en el tratamiento de los datos, conforme a lo establecido en la Ley Orgánica 15/1999, de 13 de diciembre, de Protección de Datos de Carácter Personal.

Los datos extraídos del SIGAD se han cotejado con los datos de las estadísticas mensuales del SAAD, disponibles en la Web del Instituto de Mayores y Servicios Sociales (IMSERSO) ${ }^{9}$. En nuestro estudio se tomaron como referencia los datos estadísticos a 31 de diciembre de 2013.

Después de operativizar las variables, y tras realizar las codificaciones correspondientes, se realizó el vaciado de los datos desde el 12 de enero de 2014 al 15 de febrero del mismo año, empleando para tal cometido el programa PASW Statistic SPSS 18. Del mismo modo, empleamos las tablas y representaciones gráficas que consideramos de interés, con el fin de dar respuesta a los objetivos establecidos en la investigación facilitando, de este modo, una mejor interpretación de los datos obtenidos.

\section{Resultados}

Implantación de la PEAP en Galicia. Análisis comparativo con el resto de Comunidades Autónomas del Estado desde la entrada en vigor de la LAPAD

Según la información estadística del SAAD, a fecha 31 de diciembre de 2013, nuestro país cuenta con un total de 1.644 .284 personas solicitantes

8. Disponible en http://www.ige.eu/web/mostrar_actividade_estatistica.jsp?idioma=gl\& codigo $=0201001001$ (08 Oct. 2014).

9. Disponible en http://www.dependencia.imserso.es/dependencia_01/documentacion/ estadisticas/index.htm (08 Oct. 2014) 
del reconocimiento de dependencia. De ellas, tienen derecho a prestación 944.345 personas a las que les ha sido reconocido un grado III, grado II o grado $\mathrm{I}^{10}$. En esta fecha, el número de beneficiarios con prestaciones efectivas asciende a un total de 753.842 personas (un $79,83 \%$ del total de las personas con derecho a acceder al SAAD).

Analizando la totalidad de prestaciones reconocidas (tabla 1) nos encontramos que el servicio o prestación con mayor porcentaje de resolución es la prestación económica para cuidados familiares $(43,21 \%)$, seguida del servicio de atención residencial (13,80\%), el servicio de ayuda a domicilio (12,80\%), el servicio de teleasistencia $(12,75 \%)$, las prestaciones económicas vinculadas a servicios $(7,62 \%)$, los servicios de centro de día/noche $(7,44 \%)$ y los servicios de prevención de la dependencia y promoción de la autonomía personal $(2,22 \%)$.

Del Catálogo de servicios y prestaciones que se reconocen dentro del SAAD, la PEAP ocupa el porcentaje menor de todas las reconocidas en el conjunto del Estado. Del total de prestaciones reconocidas $(945.051)^{11}$, la PEAP ha sido considerada por los técnicos competentes como la modalidad de atención más idónea, en un total de 1.441 casos; representa solamente el 0,15\% del conjunto de prestaciones. Es destacable, además, que sólo 11 de las 17 Comunidades Autónomas (CCAA) tienen reconocidas PEAP: Andalucía (14); Asturias (3); Castilla y León (56); Castilla-La Mancha (7); Catalunya (16); Comunitat Valenciana (3); Galicia (47); Madrid (23); Murcia (1); Navarra (1); País Vasco (1.270). En el resto de CCAA y en las ciudades autónomas de Ceuta y Melilla no constan resoluciones de PEAP.

Queremos subrayar las PEAP reconocidas en el País Vasco, 1.270 beneficiarios, que suponen, en esta comunidad autónoma, un 2,52\% del total de prestaciones. El número de casos en el País Vasco dista mucho de la segunda comunidad con más PEAP reconocidas, Castilla y León, con 56 personas beneficiarias.

En la comunidad autónoma de Galicia, la PEAP representa un total de 47 personas beneficiarias, lo que supone un $0,11 \%$ de la totalidad de prestaciones reconocidas (tabla 1). Aún siendo un dato elevado, en comparación con los datos del resto de CCAA, los datos extraídos de SIGAD (tabla 2) mejoran

10. En relación al calendario de implantación de la LAPAD tienen derecho a prestación aquellas personas con reconocimiento grado I nivel 2 con resolución dictada en el año 2011 y a los que se les haya reconocido la concreta prestación (Disposición final primera, LAPAD).

11. Es necesario diferenciar el número de personas atendidas y, por lo tanto, beneficiarias de prestaciones (en este caso 753.842) del número de prestaciones reconocidas (en este caso 945.051). Como puede observarse en la tabla 1, la ratio total es de 1,25 prestaciones por persona. 


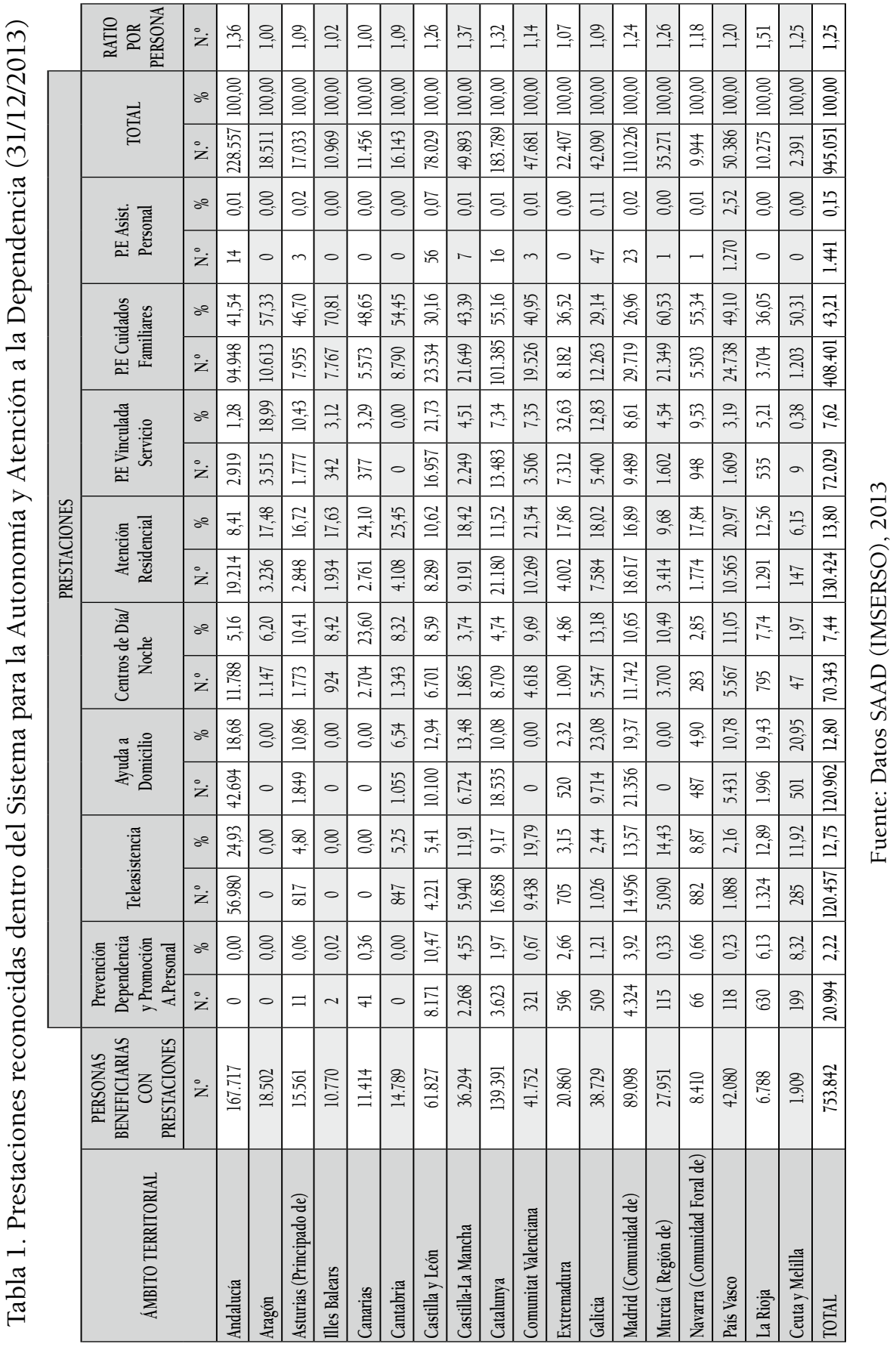

Alternativas. Cuadernos de Trabajo Social, 21, 2014, pp. 119-141 - ISSN 1133-0473 DOI: 10.14198/ALTERN2014.21.06 
Tabla 2a. Perfil de la persona beneficiaria de la PEAP según las diferentes variables y la fecha de resolución de su PIA

\begin{tabular}{|c|c|c|c|c|c|c|c|c|c|}
\hline & \multicolumn{8}{|c|}{ Resolución PIA } \\
\hline & & \multicolumn{2}{|c|}{2007} & \multicolumn{2}{|c|}{2008} & \multicolumn{2}{|c|}{2009} & \multicolumn{2}{|c|}{2010} \\
\hline & & Recuento & Porcentaje & Recuento & Porcentaje & Recuento & Porcentaje & Recuento & Porcentaje \\
\hline \multirow{3}{*}{ Sexo } & Hombre & 0 & $0,0 \%$ & 0 & $0,0 \%$ & 0 & $0,0 \%$ & 17 & $43,6 \%$ \\
\hline & Mujer & 0 & $0,0 \%$ & 0 & $0,0 \%$ & 1 & $100,0 \%$ & 22 & $56,4 \%$ \\
\hline & Total & 0 & $0,0 \%$ & 0 & $0,0 \%$ & 1 & $100,0 \%$ & 39 & $100,0 \%$ \\
\hline \multirow{6}{*}{ Edad } & $<18$ & 0 & $0,0 \%$ & 0 & $0,0 \%$ & 0 & $0,0 \%$ & 0 & $0,0 \%$ \\
\hline & 18 a 35 & 0 & $0,0 \%$ & 0 & $0,0 \%$ & 1 & $100,0 \%$ & 15 & $38,5 \%$ \\
\hline & 36 a 50 & 0 & $0,0 \%$ & 0 & $0,0 \%$ & 0 & $0,0 \%$ & 14 & $35,9 \%$ \\
\hline & 51 a 64 & 0 & $0,0 \%$ & 0 & $0,0 \%$ & 0 & $0,0 \%$ & 9 & $23,1 \%$ \\
\hline & $>=65$ & 0 & $0,0 \%$ & 0 & $0,0 \%$ & 0 & $0,0 \%$ & 1 & $2,6 \%$ \\
\hline & Total & 0 & $0,0 \%$ & 0 & $0,0 \%$ & 1 & $100,0 \%$ & 39 & $100,0 \%$ \\
\hline \multirow{8}{*}{ Ayuntamiento } & $<=2000$ & 0 & $0,0 \%$ & 0 & $0,0 \%$ & 0 & $0,0 \%$ & 1 & $2,6 \%$ \\
\hline & 2001 a 5000 & 0 & $0,0 \%$ & 0 & $0,0 \%$ & 0 & $0,0 \%$ & 4 & $10,3 \%$ \\
\hline & $\begin{array}{l}5001 \mathrm{a} \\
10000\end{array}$ & 0 & $0,0 \%$ & 0 & $0,0 \%$ & 0 & $0,0 \%$ & 3 & $7,7 \%$ \\
\hline & $\begin{array}{l}10001 \mathrm{a} \\
20000 \\
\end{array}$ & 0 & $0,0 \%$ & 0 & $0,0 \%$ & 0 & $0,0 \%$ & 9 & $23,1 \%$ \\
\hline & $\begin{array}{l}20001 \mathrm{a} \\
50000\end{array}$ & 0 & $0,0 \%$ & 0 & $0,0 \%$ & 0 & $0,0 \%$ & 8 & $20,5 \%$ \\
\hline & $\begin{array}{l}50001 \mathrm{a} \\
100000\end{array}$ & 0 & $0,0 \%$ & 0 & $0,0 \%$ & 1 & $100,0 \%$ & 4 & $10,3 \%$ \\
\hline & $\begin{array}{c}100001 \mathrm{a} \\
500000\end{array}$ & 0 & $0,0 \%$ & 0 & $0,0 \%$ & 0 & $0,0 \%$ & 10 & $25,6 \%$ \\
\hline & Total & 0 & $0,0 \%$ & 0 & $0,0 \%$ & 1 & $100,0 \%$ & 39 & $100,0 \%$ \\
\hline \multirow{5}{*}{ Provincia } & A Coruña & 0 & $0,0 \%$ & 0 & $0,0 \%$ & 0 & $0,0 \%$ & 22 & $56,4 \%$ \\
\hline & Lugo & 0 & $0,0 \%$ & 0 & $0,0 \%$ & 1 & $100,0 \%$ & 4 & $10,3 \%$ \\
\hline & Ourense & 0 & $0,0 \%$ & 0 & $0,0 \%$ & 0 & $0,0 \%$ & 2 & $5,1 \%$ \\
\hline & Pontevedra & 0 & $0,0 \%$ & 0 & $0,0 \%$ & 0 & $0,0 \%$ & 11 & $28,2 \%$ \\
\hline & Total & 0 & $0,0 \%$ & 0 & $0,0 \%$ & 1 & $100,0 \%$ & 39 & $100,0 \%$ \\
\hline \multirow{7}{*}{$\begin{array}{c}\text { Grado } \\
\text { dependencia }\end{array}$} & III 2 & 0 & $0,0 \%$ & 0 & $0,0 \%$ & 1 & $100,0 \%$ & 10 & $25,6 \%$ \\
\hline & III 1 & 0 & $0,0 \%$ & 0 & $0,0 \%$ & 0 & $0,0 \%$ & 19 & $48,7 \%$ \\
\hline & III & 0 & $0,0 \%$ & 0 & $0,0 \%$ & 0 & $0,0 \%$ & 0 & $0,0 \%$ \\
\hline & II 2 & 0 & $0,0 \%$ & 0 & $0,0 \%$ & 0 & $0,0 \%$ & 7 & $17,9 \%$ \\
\hline & II 1 & 0 & $0,0 \%$ & 0 & $0,0 \%$ & 0 & $0,0 \%$ & 3 & $7,7 \%$ \\
\hline & $\mathrm{I} 2$ & 0 & $0,0 \%$ & 0 & $0,0 \%$ & 0 & $0,0 \%$ & 0 & $0,0 \%$ \\
\hline & Total & 0 & $0,0 \%$ & 0 & $0,0 \%$ & 1 & $100,0 \%$ & 39 & $100,0 \%$ \\
\hline \multirow{4}{*}{ Tipo Tabla } & General & 0 & $0,0 \%$ & 0 & $0,0 \%$ & 0 & $0,0 \%$ & 10 & $25,6 \%$ \\
\hline & Especifica & 0 & $0,0 \%$ & 0 & $0,0 \%$ & 0 & $0,0 \%$ & 0 & $0,0 \%$ \\
\hline & No Consta & 0 & $0,0 \%$ & 0 & $0,0 \%$ & 1 & $100,0 \%$ & 29 & $74,4 \%$ \\
\hline & Total & 0 & $0,0 \%$ & 0 & $0,0 \%$ & 1 & $100,0 \%$ & 39 & $100,0 \%$ \\
\hline
\end{tabular}

Fuente: Elaboración propia. SIGAD, 2014. 
Tabla 2b. Perfil de la persona beneficiaria de la PEAP según las diferentes variables y la fecha de resolución de su PIA

\begin{tabular}{|c|c|c|c|c|c|c|c|c|c|}
\hline & & \multicolumn{8}{|c|}{ Resolución PIA } \\
\hline & & \multicolumn{2}{|c|}{2011} & \multicolumn{2}{|c|}{2012} & \multicolumn{2}{|c|}{2013} & \multicolumn{2}{|c|}{ Total } \\
\hline & & Recuento & Porcentaje & Recuento & Porcentaje & Recuento & Porcentaje & Recuento & Porcentaje \\
\hline \multirow{3}{*}{ Sexo } & Hombre & 1 & $25,0 \%$ & 2 & $50,0 \%$ & 10 & $66,7 \%$ & 30 & $47,6 \%$ \\
\hline & Mujer & 3 & $75,0 \%$ & 2 & $50,0 \%$ & 5 & $33,3 \%$ & 33 & $52,4 \%$ \\
\hline & Total & 4 & $100,0 \%$ & 4 & $100,0 \%$ & 15 & $100,0 \%$ & 63 & $100,0 \%$ \\
\hline \multirow{6}{*}{ Edad } & $<18$ & 0 & $0,0 \%$ & 0 & $0,0 \%$ & 3 & $20,0 \%$ & 3 & $4,8 \%$ \\
\hline & 18 a 35 & 1 & $25,0 \%$ & 1 & $25,0 \%$ & 5 & $33,3 \%$ & 23 & $36,5 \%$ \\
\hline & 36 a 50 & 2 & $50,0 \%$ & 2 & $50,0 \%$ & 7 & $46,7 \%$ & 25 & $39,7 \%$ \\
\hline & 51 a 64 & 1 & $25,0 \%$ & 1 & $25,0 \%$ & 0 & $0,0 \%$ & 11 & $17,5 \%$ \\
\hline & $>=65$ & 0 & $0,0 \%$ & 0 & $0,0 \%$ & 0 & $0,0 \%$ & 1 & $1,6 \%$ \\
\hline & Total & 4 & $100,0 \%$ & 4 & $100,0 \%$ & 15 & $100,0 \%$ & 63 & $100,0 \%$ \\
\hline \multirow{8}{*}{ Ayuntamiento } & $<=2000$ & 0 & $0,0 \%$ & 0 & $0,0 \%$ & 1 & $6,7 \%$ & 2 & $3,2 \%$ \\
\hline & 2001 a 5000 & 0 & $0,0 \%$ & 1 & $25,0 \%$ & 2 & $13,3 \%$ & 7 & $11,1 \%$ \\
\hline & $\begin{array}{l}5001 \mathrm{a} \\
10000\end{array}$ & 1 & $25,0 \%$ & 0 & $0,0 \%$ & 0 & $0,0 \%$ & 4 & $6,3 \%$ \\
\hline & $\begin{array}{c}10001 \mathrm{a} \\
20000\end{array}$ & 2 & $50,0 \%$ & 1 & $25,0 \%$ & 1 & $6,7 \%$ & 13 & $20,6 \%$ \\
\hline & $\begin{array}{l}20001 \mathrm{a} \\
50000\end{array}$ & 0 & $0,0 \%$ & 2 & $50,0 \%$ & 1 & $6,7 \%$ & 11 & $17,5 \%$ \\
\hline & $\begin{array}{l}50001 \text { a } \\
100000 \\
\end{array}$ & 0 & $0,0 \%$ & 0 & $0,0 \%$ & 3 & $20,0 \%$ & 8 & $12,7 \%$ \\
\hline & $\begin{array}{c}100001 \mathrm{a} \\
500000\end{array}$ & 1 & $25,0 \%$ & 0 & $0,0 \%$ & 7 & $46,7 \%$ & 18 & $28,6 \%$ \\
\hline & Total & 4 & $100,0 \%$ & 4 & $100,0 \%$ & 15 & $100,0 \%$ & 63 & $100,0 \%$ \\
\hline \multirow{5}{*}{ Provincia } & A Coruña & 3 & $75,0 \%$ & 2 & $50,0 \%$ & 1 & $6,7 \%$ & 28 & $44,4 \%$ \\
\hline & Lugo & 0 & $0,0 \%$ & 0 & $0,0 \%$ & 4 & $26,7 \%$ & 9 & $14,3 \%$ \\
\hline & Ourense & 0 & $0,0 \%$ & 0 & $0,0 \%$ & 8 & $53,3 \%$ & 10 & $15,9 \%$ \\
\hline & Pontevedra & 1 & $25,0 \%$ & 2 & $50,0 \%$ & 2 & $13,3 \%$ & 16 & $25,4 \%$ \\
\hline & Total & 4 & $100,0 \%$ & 4 & $100,0 \%$ & 15 & $100,0 \%$ & 63 & $100,0 \%$ \\
\hline \multirow{7}{*}{$\begin{array}{c}\text { Grado } \\
\text { dependencia }\end{array}$} & III 2 & 1 & $25,0 \%$ & 0 & $0,0 \%$ & 3 & $20,0 \%$ & 15 & $23,8 \%$ \\
\hline & III 1 & 1 & $25,0 \%$ & 1 & $25,0 \%$ & 2 & $13,3 \%$ & 23 & $36,5 \%$ \\
\hline & III & 0 & $0,0 \%$ & 0 & $0,0 \%$ & 1 & $6,7 \%$ & 1 & $1,6 \%$ \\
\hline & II 2 & 2 & $50,0 \%$ & 3 & $75,0 \%$ & 5 & $33,3 \%$ & 17 & $27,0 \%$ \\
\hline & II 1 & 0 & $0,0 \%$ & 0 & $0,0 \%$ & 2 & $13,3 \%$ & 5 & $7,9 \%$ \\
\hline & I 2 & 0 & $0,0 \%$ & 0 & $0,0 \%$ & 2 & $13,3 \%$ & 2 & $3,2 \%$ \\
\hline & Total & 4 & $100,0 \%$ & 4 & $100,0 \%$ & 15 & $100,0 \%$ & 63 & $100,0 \%$ \\
\hline \multirow{4}{*}{ Tipo Tabla } & General & 2 & $50,0 \%$ & 1 & $25,0 \%$ & 4 & $26,7 \%$ & 17 & $27,0 \%$ \\
\hline & Específica & 1 & $25,0 \%$ & 1 & $25,0 \%$ & 5 & $33,3 \%$ & 7 & $11,1 \%$ \\
\hline & No Consta & 1 & $25,0 \%$ & 2 & $50,0 \%$ & 6 & $40,0 \%$ & 39 & $61,9 \%$ \\
\hline & Total & 4 & $100,0 \%$ & 4 & $100,0 \%$ & 15 & $100,0 \%$ & 63 & $100,0 \%$ \\
\hline
\end{tabular}

Fuente: Elaboración propia. SIGAD, 2014.

Alternativas. Cuadernos de Trabajo Social, 21, 2014, pp. 119-141 - ISSN 1133-0473

DOI: 10.14198/ALTERN2014.21.06 
este resultado, puesto que eleva el número total de casos a 63 personas beneficiarias $^{12}$. Con este último valor, Galicia sería la segunda comunidad con más PEAP reconocidas en el conjunto del Estado, solo por detrás del País Vasco.

Asimismo, es importante subrayar que las dos ayudas cuya finalidad primordial es la promoción de la autonomía (la PEAP y los servicios de prevención y promoción) son las prestaciones de menor incidencia y, por lo tanto, con menor porcentaje de resolución.

\section{Análisis descriptivo de la persona beneficiaria de la PEAP en Galicia}

Según la información estadística del SAAD, a fecha 31 de diciembre de 2013, Galicia cuenta con un total de 82.400 personas solicitantes del reconocimiento de dependencia. De ellas, tienen derecho a prestación 56.835 personas a las que les ha sido reconocido un grado III, grado II o grado I. En esta fecha, el número de beneficiarios con prestaciones efectivas, de entre los servicios o prestaciones contemplados en el catálogo, asciende a un total de 38.729 personas (un $68,14 \%$ del total de personas con derecho a acceder al SAAD, porcentaje 10 puntos inferior al del conjunto del Estado).

Analizando la totalidad de prestaciones reconocidas (tabla 1) nos encontramos que el servicio o prestación con mayor porcentaje de resolución es la prestación económica para cuidados familiares $(29,14 \%)$, seguida del servicio de ayuda a domicilio (23,08\%), el servicio de atención residencial $(18,02 \%)$, los servicios de centro de día/noche $(13,18 \%)$, las prestaciones económicas vinculadas a servicios (12,83\%), el servicio de teleasistencia $(2,44 \%)$, y los servicios de prevención de la dependencia y promoción de la autonomía personal $(1,21 \%)$.

En relación a estos datos queremos destacar que, si bien en la LAPAD se establece que la Prestación económica para cuidados familiares se considera un tipo de prestación económica que debe tener carácter excepcional de concesión, es (aún a día de hoy) el tipo de prestación más frecuente en los Programas individuales de atención resueltos. Sin embargo, el porcentaje de resolución en Galicia $(29,14 \%)$ es notablemente inferior al del conjunto del Estado (43,21\%).

Los Órganos de valoración y asesoramiento de la dependencia de la Xunta de Galicia han considerado la PEAP como la modalidad de atención más idónea para 63 casos de entre todos los beneficiarios del SAAD en ese

12. Entendemos que la divergencia entre los datos del Estado y la Comunidad Autónoma son altas de nuevos beneficiarios, registrados en la aplicación informática SIGAD, pero que todavía no se han incorporado a las base de datos del SAAD. 
territorio, en el período comprendido entre el año 2007 y 2013, ambos incluidos (tabla 2).

Sorprende, en primer término, la inexistencia de resoluciones de PEAP en los ejercicios 2007 y 2008. En el año 2009 solamente aparece una prestación reconocida, en este caso, correspondiente al Equipo de valoración de la provincia de Lugo. El número de casos fue variable a lo largo de todos los años estudiados. Es significativo que más de la mitad de los casos de PEAP (39) corresponde al año 2010. Después de estancarse en los años 2011 y 2012 con solo 4 casos por año, en el año 2013 se produce un nuevo repunte con 15 casos resueltos; a destacar el número de casos de la provincia de Ourense que suponen más del $50 \%$ en ese ejercicio.

Tras el análisis de los datos obtenidos del SIGAD, observamos que el perfil mayoritario de los beneficiarios de la PEAP en la Comunidad Autónoma de Galicia se trata de una persona cuya edad se sitúa en el rango de 18 a 50 años, no encontrándose diferencias significativas respecto al sexo (figura 1).

Figura 1. Perfil de la persona beneficiaria de la PEAP según el sexo y edad

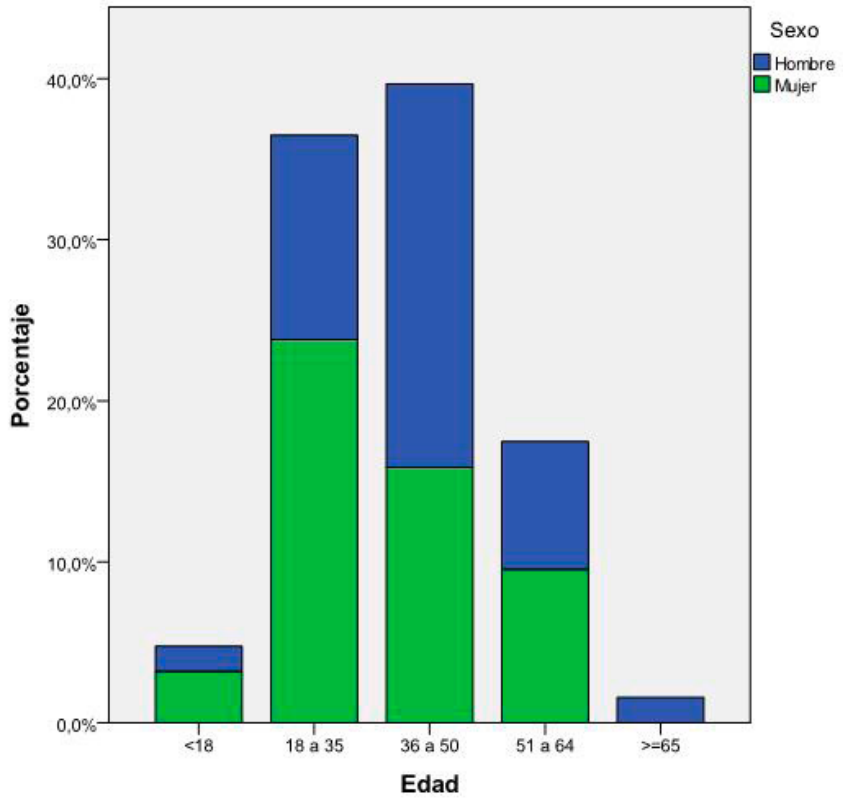

Fuente: Elaboración propia. SIGAD, 2014. 
$\mathrm{Al}$ respecto de la edad de la persona beneficiaria de la PEAP es necesario realizar unas precisiones. Al agrupar los datos en intervalos, para obtener más relevancia estadística, observamos que los rangos de edad con mayores frecuencias son las franjas de 18 a 35 años (36,5\%) y de 36 a 50 años (39,7\%); representan por lo tanto el $75,5 \%$ del total que componen la muestra. Sin embargo, queremos destacar que 3 de los sujetos tienen una edad $<18$ años y existe también un caso de un beneficiario con edad $\geq 65$ años (tabla 2).

La unidad de convivencia de la persona beneficiaria reside mayoritariamente en un entorno semi-urbano o urbano. Casi un tercio de los casos estudiados residen en poblaciones de más de 100.000 habitantes. Si agrupamos los datos podemos observar como sólo el 20,6\% de las PEAP se reconocen en localidades con población $\leq 10.000$ habitantes. Esta tendencia se mantiene a lo largo de todos los años estudiados.

Las personas beneficiarias de la PEAP son mayoritariamente grandes dependientes. Así, al 61,9\% de los sujetos estudiados se les ha reconocido una situación de gran dependencia. La dependencia severa representa el 34,9\% de los casos y la dependencia moderada un $3,2 \%$.

El Baremo de valoración de dependencia, regulado en el Real Decreto 174/2011, de 11 de febrero ${ }^{13}$, establece una tabla general en la que se recogen las diferentes Actividades de la vida diaria (divididas en tareas) que han de valorase para determinar la situación de dependencia y el grado de severidad de la misma. Asimismo establece que, en los casos en los que mediante informe de salud se documente una afectación del funcionamiento mental de la persona valorada, procederá aplicar una tabla especifica. En dicha tabla se añade a la evaluación ordinaria, la valoración de la capacidad que tiene la persona para Tomar decisiones. Pues bien, con los datos obtenidos en nuestro estudio y con respecto al tipo de tabla aplicada, no se puede determinar la relevancia de la misma, puesto que en un $61,9 \%$ de los casos (mayoritariamente correspondientes al año 2010) no constaba el tipo de tabla valorada.

Podemos apreciar, sin embargo, que solo en un $11,1 \%$ de los sujetos estudiados les fue aplicado el Baremo de valoración de dependencia mediante la tabla específica. Esto quiere decir que, en esos casos, estaba documentado

13. El baremo de valoración de dependencia recogido en el Real Decreto 504/2007, de 20 de abril, se mantuvo vigente desde el 22 de abril de 2007 al 17 de febrero de 2012, siendo en la actualidad substituido por el baremo recogido en el Real Decreto 174/2011, de 11 de febrero, por el que se aprueba el baremo de valoración de la situación de dependencia establecido en la Ley 39/2006, que deroga expresamente el Real Decreto 504/2007, y que tuvo su entrada en vigor el 18 de febrero de 2012. Los sujetos de nuestro estudio han sido valorados con alguno de los dos instrumentos recogidos en las disposiciones anteriormente referidas. 
que la persona padecía una afectación de su funcionamiento mental. La problemática de desempeño de tipo físico, en los casos constatados, representa un $27 \%$ del total.

\section{Discusión}

Vistos los resultados que acabamos de presentar, podemos afirmar que la PEAP es la modalidad de atención minoritaria de entre el conjunto de servicios y prestaciones que conforman el Catálogo del SAAD, tanto en la comunidad autónoma de Galicia como en el conjunto del Estado.

La baja frecuencia de resolución de la PEAP es una constante desde la entrada en vigor de la LAPAD. Así, tanto en datos del propio SAAD, como en los de otras investigaciones, ha quedado patente dicha circunstancia. LópezPérez (2012) realizó un estudio sobre la implantación de la PEAP hasta el año 2011 y ya constataba que el desarrollo de esta prestación resultaba insuficiente. Apuntaba principalmente a la falta de desarrollo normativo específico, la falta de presupuesto económico y también a la falta de voluntad política. Estudios más recientes a nuestro trabajo siguen constatando un bajo porcentaje de resolución de la PEAP en el marco del SAAD. En mayo de 2014, la PEAP representa un porcentaje del 0,19\% de todas las prestaciones reconocidas (Barriga, Brezmes, García y Ramírez, 2014).

Recordemos que la LAPAD establece que la PEAP tiene como objetivo promocionar la autonomía de las personas con dependencia reconocida (en cualquiera de sus grados), contribuyendo a la contratación de una asistencia personal para facilitar el acceso a la educación y al trabajo de la persona beneficiaria así como una vida más autónoma en el ejercicio de las ABVD (art. 19, LAPAD). En relación a los datos obtenidos en nuestra investigación (y también en base a nuestra experiencia en la gestión de estas prestaciones) vamos a concretar unos puntos débiles que entendemos es importante corregir para posibilitar una mayor implantación y desarrollo de la PEAP.

Restricción en relación a la severidad de la situación de la dependencia

Hasta la modificación recogida en el Real Decreto-ley 20/2012, de 13 de julio, la LAPAD establecía como requisito básico para poder beneficiarse de la PEAP el tener reconocida una situación de gran dependencia. Por lo tanto, las personas con reconocimiento de dependencia severa o moderada tenían restringido el acceso a esta prestación. Como pudimos observar en el análisis de los datos de nuestra investigación, en la comunidad autónoma de Galicia aparecen reconocimientos de PEAP a grados II en los años 2010, 2011 y 2012 
(previos a la entrada en vigor de la disposición normativa referenciada). Esto fue posible porque en Galicia, cualquier persona reconocida con el grado de dependencia severa ya podía beneficiarse de la PEAP desde el año 2010 (disposición transitoria novena, Decreto $15 / 2010)^{14}$, todo ello en función del calendario de implantación progresiva que establece la LAPAD. Esta restricción en el acceso al servicio de asistente personal por parte de las personas con dependencia severa puede justificar que en Galicia no aparezca ninguna PEAP reconocida en el ejercicio 2008 y un único caso en el año 2009.

\section{La finalidad de la prestación}

Como ya hemos apuntado previamente, el desarrollo reglamentario de la LAPAD ha seguido cursos diferentes en las distintas comunidades autónomas. Por ello, vamos a centrarnos en los requisitos exigibles para el reconocimiento de la PEAP recogidos el la propia LAPAD. La normativa vigente a nivel estatal establece como finalidad de la PEAP el facilitar el acceso a la educación y al trabajo así como una vida más autónoma en el ejercicio de las ABVD. En la introducción de este trabajo señalábamos la gran diversidad de tareas que englobaba el servicio de asistencia personal y sus múltiples posibilidades. Las funciones y atribuciones del asistente personal van mucho más allá que el mero «acompañamiento» a la escuela o al trabajo. Del mismo modo, dichas tareas se desarrollan a lo largo de todo el ciclo vital de la persona con diversidad funcional y no solamente en la edad correspondiente a las etapas educativa y laboral. No se contempla el uso de la prestación para el ocio, lo que indica una clara discriminación con el resto de la ciudadanía (Rodríguez-Picavea y Romanach, 2006). Puede entenderse, en relación a la redacción de la Ley, que ha de exigirse como requisito el estar ejerciendo una actividad formativa y/o una actividad laboral, lo que supondría a día de hoy dejar sin acceso a la PEAP a las personas $<16$ años $\mathrm{y}>65$ años.

\section{La edad}

En las disposiciones normativas estatales no está regulada una edad mínima para poder acceder a la PEAP $^{15}$. En base a las competencias que tienen atri-

14. El Decreto 15/2010, de 4 de febrero, recoge en su disposición transitoria novena que podrá concederse la PEAP en supuestos de dependencia severa cuando, a juicio de los técnicos competentes, se entienda que esta prestación económica, en función de circunstancias objetivables, es la idónea dentro del catálogo de servicios y prestaciones del SAAD.

15. El Real Decreto 1051/2013, de 27 de diciembre, dispone en su art. 11 que los requisitos de acceso a las prestaciones económicas se establecerán por la comunidad autónoma o 
buidas, las diferentes autonomías han reglamentado las condiciones de acceso para la PEAP con diferentes criterios. Así, en comunidades autónomas como el País Vasco (Benedicto, 2013) o la Comunidad Valenciana ${ }^{16}$ se establece una edad mínima de 3 años. Por el contrario, en Galicia se exige que, al menos, la persona solicitante de la prestación tenga cumplidos los 16 años de edad ${ }^{17}$. Parece totalmente arbitrario y discriminatorio el acotar los tramos de edad de las personas con diversidad funcional que puedan beneficiarse de la PEAP. En contraposición a esta limitación, en la conceptualización del servicio de asistente personal aportada por el Movimiento de vida independiente se establece que «en el caso de menores de edad y personas sin autogobierno, el asistente personal trabaja sobre un protocolo establecido con el representante legal y teniendo siempre en cuenta las opiniones de la persona con diversidad funcional hasta donde ésta pueda decidir» (Rodríguez-Picavea, 2007: 117). En los resultados de nuestra investigación parece confirmarse la dependencia de la variable edad a la hora de resolver la PEAP. Así, podemos comprobar como las franjas de edad con mayores frecuencias absolutas son el tramo de 18 a 35 y de 36 a 50 años. De la muestra de estudio (63 personas) solo se reconoció la PEAP a una persona $\geq 65$ años y a tres personas con menos de 18 años.

\section{La capacidad para tomar decisiones}

En relación a la capacidad de tomar decisiones, tampoco las disposiciones normativas han facilitado la resolución de la PEAP. Es necesario recordar que el representante legal de la persona con diversidad funcional puede complementar (lo más adecuado), o incluso suplir, la actividad de Tomar decisiones en los casos en los que la persona beneficiaria sea menor de edad o exista afectación de su capacidad mental. En un trabajo en el que efectuaba un análisis comparativo del desarrollo reglamentario de la LAPAD en diferentes comunidades autónomas en relación al servicio de asistencia personal, Rueda (2013) ya identificaba como problemático el hecho de que el servicio se limitase en general a personas con diversidad funcional física. Enfatizaba el caso de Cataluña donde se contempla también el servicio para personas con

Administración que, en su caso tenga la competencia, teniendo en cuenta los acuerdos que adopte el Consejo Territorial de Servicios Sociales y del Sistema para la Autonomía y atención a la Dependencia

16. http://www.bsocial.gva.es/web/dependencia/asistente-personal (10 Oct. 2014)

17. El Decreto 149/2013, de 5 de septiembre, establece en su art. 5.4 que forman parte de la cartera del servicio de asistente personal los servicios dirigidos a facilitar el apoyo personal, inclusión social y/o educativa, así como la promoción y participación de las personas que, siendo de edad igual o superior a 16 años (...) 
diversidad funcional sensorial y el caso de Guipúzcoa en donde no existe limitación de acceso en este sentido. En el caso de Galicia si está establecido normativamente la exclusión de acceso para las personas sin capacidad de tomar decisiones ${ }^{18}$; no se contempla, en ningún caso, que la capacidad de dar ordenes y supervisar el servicio de asistencia personal pueda estar delegado en la persona que representa a la persona con diversidad funcional. En nuestro trabajo parece comprobarse dicha limitación; de este modo, en solo 7 de los 63 casos estudiados aparece valorada la actividad Tomar decisiones del Baremo de valoración de dependencia.

Interferencia de la prestación económica para cuidados en el entorno familiar

La coyuntura económica actual, que supone que en muchas unidades convivenciales exista una disminución importante de los ingresos familiares, ha significado (entre otros factores) la preferencia de las personas solicitantes por una prestación concreta del catálogo del SAAD: la prestación económica por cuidados familiares (recordemos que representa el 43,21\% del conjunto de prestaciones reconocidas). A ello se añade el interés de las Administraciones por resolver prestaciones de menor cuantía económica que supone, por ejemplo, que con la cuantía correspondiente a una PEAP puedan casi resolverse dos prestaciones de cuidados familiares ${ }^{19}$. Sin duda, esta determinación (tanto de los propios solicitantes como de las Administraciones públicas) ha significado que muchas atenciones de asistencia personal, que se realizan de facto, queden encubiertas en el reconocimiento de la prestación de cuidados familiares. Como señalábamos en nuestro análisis de datos, País Vasco es la comunidad autónoma con más PEAP reconocidas. En un estudio realizado en la provincia de Guipúzcoa se ha determinado que de las 923 solicitudes de PEAP registradas hasta noviembre de 2013, 310 eran «migraciones» de prestaciones económicas de cuidado en el entorno a prestaciones de asistencia personal (Benedicto, 2013). Sin duda, potenciar que este tipo de situaciones

18. La Orden de desarrollo del Decreto 15/2010 (2 de enero de 2012), establece los requisitos para acceder a la PEAP en el marco del SAAD. En su art. 42.1.b dispone como requisito especifico que la persona en situación de dependencia esté capacitada para ordenar y supervisar las actividades del servicio de asistencia personal.

19. El Real Decreto 1051/2013, de 27 de diciembre, establece en su disposición adicional segunda que las cuantías máximas de las prestaciones económicas serán las que se determinan en la disposición transitoria décima, punto 2, del Real Decreto-ley 20/2012, de 13 de julio. De este modo, la cuantía máxima (correspondiente al grado III de dependencia) para la PEAP se fija en $715,07 € /$ mes y para la prestación de cuidados familiares en $387,64 € /$ mes. 
afloren, y consten como tal en todas las estadísticas oficiales, potenciará la visualización social de la PEAP por parte de toda la ciudadanía.

\section{El impulso por parte de las Administraciones Públicas}

En relación al desarrollo e implantación de la LAPAD Montalbá (2013) identificaba claramente problemas de coordinación interadministrativa y falta de liderazgo de la Administración General del Estado para homogeneizar la implantación de los diferentes servicios y prestaciones en todo el territorio; concluía con que podían constatarse numerosos ejemplos de mal gobierno. Asimismo, López-Pérez (2012) apuntaba a la falta de voluntad política en relación a potenciar la implantación de la PEAP, afirmaciones ambas que compartimos plenamente. En un trabajo sobre la que denominaba Ley de la «Independencia», Arnau (2006) recogía ejemplos de buenas prácticas en relación al impulso de los servicios de asistencia personal. Se refería a tres iniciativas por parte de las Administraciones públicas (Diputación Foral de Guipúzcoa, Comunidad de Madrid y Ayuntamiento de Barcelona) que impulsaban tres proyectos pilotos de vida independiente. A ellos queremos añadir el Proyecto Piloto de Asistencia Personal para personas con discapacidad y gran dependencia que promovió la Vicepresidencia de la Igualdad y el Bienestar (Xunta de Galicia) a través de la Confederación Gallega de personas con discapacidad, COGAMI (Campello y Tubío, 2008). A la vista de los resultados obtenidos, parece que este intervencionismo por parte de las Administraciones ha significado una mayor implantación de la PEAP. Las comunidades autónomas de País Vasco, Galicia, Cataluña y Madrid ocupan, junto a Castilla y León, los primeros puestos según los porcentajes de resolución de las PEAP. En el caso concreto de Galicia, las personas que participaron en este proyecto piloto se incluyeron dentro del SAAD, como beneficiarias de la PEAP, en el año 2010 (39 casos). Al mismo tiempo, posibilitaron la modificación normativa, ya señalada, recogida en el Decreto 15/2010 para que las personas con dependencia severa también pudieran beneficiarse de la PEAP.

\section{Desconocimiento del servicio de asistente personal}

Sin disponer de ningún dato objetivable que lo sustente, queremos añadir a los factores referidos previamente un nuevo elemento que constatamos a diario a través de nuestra experiencia en la gestión: el desconocimiento del servicio de asistencia personal. A diario nos encontramos con personas solicitantes, familiares y también con personal técnico, tanto del ámbito social como sanitario, que desconocen en qué consiste el servicio de asistencia 
personal y las tareas que desarrolla un asistente personal. Es frecuente que se confunda el servicio de asistente personal con las funciones que lleva a cabo el personal auxiliar del Servicio de ayuda a domicilio y también con los cuidados informales. En nuestra investigación se constata claramente un mayor índice de resoluciones de PEAP en entornos urbanos y semi-urbanos; sin duda, donde las personas tienen un mayor nivel educativo y también más acceso a todos los diferentes servicios de uso público. En relación tanto a este factor, como al señalado previamente a éste, entendemos que debe prestarse (desde la Administración y desde los colectivos que representan a las personas con diversidad funcional) más información, publicidad y formación a los técnicos del sistema de servicios sociales y del sistema sanitario que garantice el conocimiento de la PEAP e impulse su prescripción como servicio idóneo en muchos de los casos valorados.

En resumen, el presente trabajo posibilita un mayor conocimiento de la atención y de las características de la PEAP, acercándonos un poco más al perfil de la persona beneficiaria de dicha prestación. Del mismo modo, identifica una serie de factores que entendemos dificultan el desarrollo de esta prestación y la implantación efectiva dentro del SAAD, y que es necesario corregir. Si bien los resultados apoyan que la PEAP es, a día de hoy, una prestación minoritaria, queremos resaltar la importancia del servicio de asistencia personal en beneficio de las personas con diversidad funcional (de cualquier tipo) para alcanzar la igualdad y la integración en la sociedad en condiciones equiparables al resto de la ciudadanía.

\section{Bibliografía}

Aguado, A.L. (1995). Historia de las deficiencias. Madrid: Escuela Libre Editorial. ARNAU, M.S. (2004). Sexualidad(es) y disCapacidad(es): La igualdad diferente. Ponencia presentada en el Seminario Discapacidad y Vida Independiente, Universidad Internacional Menéndez Pelayo e Instituto de Migraciones y Servicios Sociales, Santander, España.

ARNAU, M.S. (2006). Ley de la «In-dependencia»: pasos para garantizar un nuevo derecho de ciudadanía. Lan harremanak: Revista de relaciones laborales, 15, 41-64.

BarRiga, L.A., Brezmes, M.J., García, G.A., y RAMírez, J.M. (2014). Informe sobre el desarrollo y evaluación territorial de la ley de promoción de la autonomía personal y atención a las personas en situación de dependencia. Julio 2014. http://www.directoressociales.com/documentos/dictamenes-observatorio.html/ (10 Oct. 2014). 
BENEDicto, Z. (2013). La prestación económica de asistencia personal en el territorio histórico de Gipuzkoa. Zerbitzuan, 54, 67-84. http://dx.doi. org/10.5569/1134-7147.54.04

CAmpello, L. y Tubío, J. (2008). Proxecto piloto de asistencia persoal: estudo e avaliación. Santiago de Compostela: COGAMI.

CAmpo, M., Crespo, M., y Verdugo, M.A. (2003). Historia de la clasificación internacional del funcionamiento, de la discapacidad y de la salud (CIF). Siglo Cero: Revista Española sobre Discapacidad Intelectual, 34(I), 20-26.

Casado, D. (2001). Conceptos sobre la discapacidad. Apuntes. Boletín del Real Patronato sobre Discapacidad, 50, 5-14.

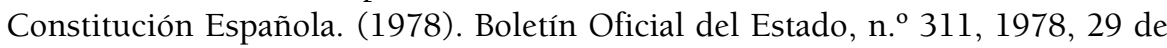
diciembre.

Decreto 15/2010, por el que se regula el procedimiento para el reconocimiento de la situación de dependencia y del derecho a las prestaciones del sistema para la autonomía y atención a la dependencia, el procedimiento para la elaboración del Programa Individual de Atención y la organización y funcionamiento de los órganos técnicos competentes. (2010, 4 de febrero). Diario Oficial de Galicia, n. ${ }^{\circ}$ 34, 2010, 19 de febrero.

Decreto 149/2013, por el que se define la cartera de servicios sociales para la promoción de la autonomía personal y la atención a las personas en situación de dependencia y se determina el sistema de participación de las personas usuarias en el financiamiento de su coste. (2013, 5 de septiembre). Diario Oficial de Galicia, n. ${ }^{\circ}$ 182, 2013, 24 de septiembre.

GARCíA, J.L. (1990). Sexualidad y deficiencia. Minusval, 69, 15-17.

García, J.V. (Coord.) (2003). El movimiento de vida independiente: experiencias internacionales. Madrid: Fundación Luis Vives.

IÁÑEZ, A. (2009). Prisioneros del cuerpo: la construcción social de la diversidad funcional. Santiago de Compostela: Obra Social Caja Madrid.

Ley 39/2006 de Promoción de la Autonomía Personal y Atención a las personas en situación de dependencia. (2006, 14 de diciembre). Boletín Oficial del Estado, n. ${ }^{\circ} 299,2006,15$ de diciembre.

Ley Orgánica 15/1999, de Protección de Datos de Carácter Personal. (1999, 13 de diciembre). Boletín Oficial del Estado, n. ${ }^{\circ}$ 298, 1999, 14 de diciembre.

LÓPEZ-PÉREZ, M. (2012). Prestación económica de asistencia personal: asignatura pendiente de la Ley 39/2006, de 14 de diciembre. Portularia, 12(Extra), 111-120.

LuJÁn, J., RodríGUEZ, G., y FERnÁNDEZ, FJ. (2008). El sistema integral de promoción de la autonomía personal y atención a las personas en situación de dependencia. Las prestaciones. En A.V. Sempere (Dir.). Comentario sistemático a la Ley de la Dependencia (283-323). Navarra: Thomson-Aranzadi. 
MEDinA, M.B. (2010). Evaluación de la conducta adaptativa de las personas con discapacidad intelectual. Valoración y usos de la Escala ABS-RC:2. Tesis doctoral, Universidad de Burgos, Burgos, España. http://dspace.ubu.es:8080/tesis/ bitstream/10259/101/1/Medina_G\%C3\%B3mez.pdf (10 Oct. 2014).

Montalbá, C. (2013). Aplicación del sistema de promoción de la autonomía personal y atención a las personas en situación de dependencia en la Comunitat Valenciana: efectos sobre el ejercicio del derecho social. Alternativas Cuadernos de trabajo social, 20, 163-186.

Orden de desarrollo del Decreto 15/2010, de 4 de febrero, por el que se regula el procedimiento para el reconocimiento de la situación de dependencia y del derecho a las prestaciones del Sistema para la Autonomía y Atención a la Dependencia, el procedimiento para la elaboración del programa individual de atención y la organización y funcionamiento de los órganos técnicos competentes. (2012, 2 de enero). Diario Oficial de Galicia, n. ${ }^{\circ}$ 9, 2012, 13 de enero.

Organización Mundial de la Salud (2001). Clasificación Internacional del Funcionamiento, de la Discapacidad y de la Salud. Madrid: IMSERSO.

Real Decreto 174/2011 por el que se aprueba el baremo de valoración de la situación de dependencia establecido por la Ley 39/2006, de 14 de diciembre, de Promoción de la Autonomía Personal y Atención a las personas en situación de dependencia. (2011, 11 de febrero). Boletín Oficial del Estado, n. ${ }^{\circ} 42$, 2011, 18 de febrero.

Real Decreto 504/2007 por el que se aprueba el baremo de valoración de la situación de dependencia establecido por la Ley 39/2006, de 14 de diciembre, de Promoción de la Autonomía Personal y Atención a las personas en situación de dependencia. (2007, 20 de abril). Boletín Oficial del Estado, n. o 96, 2007, 21 de abril.

Real Decreto 1051/2013 por el que se regulan las prestaciones del Sistema para la Autonomía y Atención a la Dependencia, establecidas en la Ley 39/2006, de 14 de diciembre, de Promoción de la Autonomía Personal y Atención a las personas en situación de dependencia. (2013, 27 de diciembre). Boletín Oficial del Estado, n. ${ }^{\circ} 313,2013,31$ de diciembre.

Real Decreto-ley 20/2012, de medidas para garantizar la estabilidad presupuestaria y fomento de la competitividad. (2012, 13 de julio). Boletín Oficial del Estado, n. ${ }^{\circ}$ 168, 2012, 14 de julio.

RodríGUEZ-PiCAVEA, A. (2007). Reflexiones en torno a la figura del asistente personal para la vida independiente y la promoción de la autonomía de las personas con diversidad funcional (discapacidad). Zerbitzuan, 41, 115-128.

Rodríguez-PicaVeA, A., y RomanaCH, J. (2006). Consideraciones sobre la figura del Asistente Personal en el Proyecto de la Ley de Promoción de la Autonomía 
Personal y Atención a las Personas en Situación de Dependencia. www.carm. es/ctra/cendoc/haddock/14123.pdf (10 Oct. 2014).

RuEDA, M. (2013). Análisis comparativo de las iniciativas de vida independiente en España. http://www.forovidaindependiente.org (10 Oct. 2014).

Sempere, A.V. (2008). Presupuestos, antecedentes y gestación de la Ley. En A.V. Sempere (Dir.). Comentario sistemático a la Ley de la Dependencia (77-112). Navarra: Thomson-Aranzadi.

Sexualité des personnes âgées et handicapées: la fin d'un tabou?. (2010). Actualités sociales hebdomadaires, 2671, 38-41.

United Nations (2006). Convención sobre los derechos de las personas con discapacidad. $h t t p: / / w w w . u n . o r g / e s a / s o c d e v / e n a b l e / d o c u m e n t s / t c c c o n v s . p d f$ (10 Oct. 2014). 